\title{
Carbon superatom thin films
}

\author{
Andrew Canning ${ }^{(a, b)}$, Giulia Galli ${ }^{(b, *)}$ and Jeongnim $\operatorname{Kim}^{(c)}$ \\ (a) Cray Research, PSE, EPFL, 1015 Lausanne, Switzerland. \\ (b) Institut Romand de Recherche Numérique en Physique des Matériaux (IRRMA), IN-Ecublens, \\ 1015 Lausanne, Switzerland. \\ (c) Department of Physics, The Ohio State University, Columbus OH 43210, USA. ${ }^{\dagger}$
}

\begin{abstract}
Assembling clusters on surfaces has emerged as a novel way to grow thin films with targeted properties. In particular, it has been proposed from experimental findings that fullerenes deposited on surfaces could give rise to thin films retaining the bonding properties of the incident clusters. However the microscopic structure of such films is still unclear. By performing quantum molecular dynamics simulations, we show that $\mathrm{C}_{28}$ fullerenes can be deposited on a surface to form a thin film of nearly defect free molecules, which act as carbon superatoms. Our findings help clarify the structure of disordered small fullerene films and also support the recently proposed hyperdiamond model for solid $\mathrm{C}_{28}$.
\end{abstract}


A growing community of physicists, chemists and material scientists have recently devoted a significant effort to studying and controlling the behaviour of small clusters, the main goal being the synthesis of new materials [1]. In particular, assembling clusters by deposition on surfaces is emerging as a promising way to grow thin films with targeted properties. Since the experimental discovery of $\mathrm{C}_{60}$ [2], many investigations have focussed on fullerenes, due to the variety of exciting properties of pure and doped systems in both the gas and solid phases.

Recent experiments [3] suggest that low energy deposition of fullerenes on semiconducting substrates can produce thin films which retain the bonding properties of the incident clusters. This memory effect might permit the synthesis of amorphous diamond or graphitic-like films depending on whether small $\left(\mathrm{C}_{\mathrm{n}}, \mathrm{n}=20-32\right)$ or large $\left(\mathrm{C}_{900}\right)$ clusters are deposited [3]. Of all the small fullerenes, $\mathrm{C}_{28}$ is the most studied: this molecule is believed to be the smallest

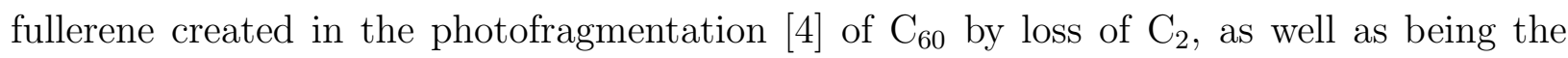
smallest fullerene produced in considerable amounts in the laser vapourisation of graphite [5]. The structure of the isolated cluster was first proposed by Kroto [6], who suggested that $\mathrm{C}_{28}$ should be chemically very reactive, with four preferred active sites. Recently, the existence of solid forms of $\mathrm{C}_{28}$ has been conjectured. Guo et al. [5] proposed that it should be possible to stabilise an arrangement of the fullerene cages in a diamond lattice (hyperdiamond structure). Since then several studies of various ordered $\mathrm{C}_{28}$ solids have appeared in the literature [7 [10], however, the possible growth and stability of $\mathrm{C}_{28}$ solids as well as the ability of the clusters to form thin films remain at present open questions.

In order to address these issues, we performed quantum molecular dynamics simulations to investigate the deposition process of $\mathrm{C}_{28}$ cages on a non-metallic substrate, corresponding to the early stages of the growth of a disordered thin film. We used a tight-binding model [11] in the context of a linear scaling method [12 [14]. Here we present an analysis of the deposition process, we discuss the microscopic structural properties of the deposited film and relate them to recent experimental findings [3,5]. 
In our simulations, the interatomic potential is derived from the total energy of interacting ions and electrons, as described by a tight-binding (TB) model [11]. This model has been successfully used to study a variety of carbon systems [11. We chose a TB picture rather than a classical potential since the high reactivity of $\mathrm{C}_{28}$ clusters and their ability to form covalent bonds, call for a quantum mechanical treatment of the interactions. As we will justify, a TB picture is appropriate for the purpose of this study. We performed simulations with cells of up to 4472 atoms, for several tens of picoseconds. These calculations of unprecedented size were made possible by the favourable scaling with system size of the algorithm that we adopted, together with its efficient implementation on a massively parallel computer [14].

The validity and accuracy of linear scaling $[\mathrm{O}(N)]$ methods for systems similar to those studied in the present work have been addressed in several recent publications [11-13, 15]. In order to test the validity of our $\mathrm{O}(\mathrm{N})$ - TB model for the description of $\mathrm{C}_{28}$ systems, we compared results [16] for the $\mathrm{C}_{28}$ monomer, dimer and hyperdiamond ordered solid with those of first principles local density (LDA) functional calculations. The comparison is reported in the Table and shows that the agreement between LDA and $\mathrm{O}(\mathrm{N})$-TB results is extremely good. We therefore conclude that the $\mathrm{O}(\mathrm{N})-\mathrm{TB}$ approach is a sufficiently accurate model to describe inter- and intra- $\mathrm{C}_{28}$ interactions.

In our computer experiment [16,17], we projected 78 fullerenes onto a diamond substrate, of which 50 remained to form a film (see Fig. 1). The substrate consisted of 12 C layers with 256 atoms per layer with periodic boundary conditions in the $x$ and $y$ (horizontal) directions, and a $(2 \times 1)$ reconstructed $(111)$ surface on the upper and lower side. The deposition was performed by sending down 14 separate showers of $\mathrm{C}_{28}$ clusters: the initial configuration of each shower was prepared by choosing molecules randomly distributed in space and noninteracting with each other and with the surface (due to the localisation of the orbitals). They were also chosen to have random orientations. The number of fullerenes belonging to each shower varied from 4 to 8 depending on the maximum number of noninteracting molecules whose center of mass could be randomly placed on a plane parallel to, 
and of the same size as the surface layer. With a series of test runs (involving the study of fullerene-fullerene and fullerene-surface impacts as a function of energy), we determined the range of incident kinetic energies $(20-40 \mathrm{eV})$ for which the fullerenes had the highest sticking probability without breaking up. We then projected each shower, one after the other, vertically downwards onto the surface, with each molecule having an initial kinetic energy randomly distributed within this range of energies. In this way we tried to mimic some of the features of low-energy neutral-cluster beam deposition (LECBD) experiments [3]. The bottom three layers of the substrate were held fixed and the next three layers were connected to a Nosé-Hoover thermostat [18 at room temperature to prevent the substrate heating up [19] under the many fullerene impacts. After each shower of fullerenes the whole system was equilibrated, by monitoring the variation in time of the number of bonds as well as the bond lengths.

At the end of the deposition process, 24 fullerenes were bonded directly to the substrate, with the majority of them sticking to the surface during the first 3-4 showers. The arrangement of the first monolayer of $\mathrm{C}_{28}$ 's on the surface is random, with no preferential bonding sites (see Fig. 2a). As expected from the large number of dangling bonds in the cluster, $\mathrm{C}_{28}$ 's easily attach to the diamond substrate, the average number of bonds with the surface being about three per fullerene. These can be classified as strong covalent bonds: their formation can cause bonds between the fourfold coordinated atoms of the first and second surface bilayer to break. This locally destroys the $(2 \times 1)$ Pandey chain reconstruction, returning the surface geometry to an ideal (111) configuration (de-reconstruction). Nevertheless the formation of bonds between the substrate and $\mathrm{C}_{28}$ 's does not induce modifications of their molecular topology. This is at variance to what was observed for $\mathrm{C}_{60}$ on a diamond (111) surface [20], where impacts leading to bonds between the $\mathrm{C}_{60}$ and the surface would necessarily induce the formation of defects in the cage, which could lead to its break up in the high energy regime 20].

On the time scale of our simulation we did not observe any diffusion of the $\mathrm{C}_{28}$ 's on the surface. Most importantly, when heating the system after the deposition was completed, no 
diffusion was observed before reaching the temperature regime where cages started to break up. Therefore, we expect that the bonds between the fullerenes and the surface are strong enough to hamper rearrangements and diffusion. Consequently, the system is unlikely to undergo a disorder to order transition like the one observed experimentally in the case of $\mathrm{C}_{60}$ on some semiconducting substrates [21]. Indeed, for $\mathrm{C}_{60}$, van der Waals interactions between the molecules and the substrate play a crucial role in allowing cluster diffusion and the subsequent disorder to order transition.

On top of the first monolayer, a second portion of the system (26 fullerenes) was identified, which was again very inhomogeneous. In this portion of the system, during the deposition process, we observed the formation of three-dimensional islands with some polymer-like structures, where $\mathrm{C}_{28}$ 's were preferentially bonded to each other with two single bonds (see Fig. 2b and 2c). This is suggestive of either a Stranski-Krastanov or a Volmer-Weber growth process [22]. However our simulation cannot clearly establish a growth process mechanism since larger cell sizes and longer simulation times would be needed [23]. After equilibration at room temperature, the film was heated up to verify its thermal stability. During the heating procedure, we observed oscillations of the polymer-like structures with no major structural change. Our results indicate that the film is stable up to about $1000 \mathrm{~K}$ [23].

The whole system at the end of the deposition process at room temperature is shown in Fig. 1. The thin film is characterised by fullerenes which retained their topology, with small distortions 24] with respect to the original shapes. These results are in agreement with the memory effect reported experimentally when depositing small fullerenes by LECBD [3]. Furthermore in our deposited film the particle density is very low $\left(\simeq 1 \mathrm{gr} \mathrm{cm}^{-3}\right.$, compared to the diamond and graphite densities of 3.52 and $2.27 \mathrm{gr}^{-3}$, respectively) consistent with the measured value of $0.8 \pm 0.2 \mathrm{gr} \mathrm{cm}^{-3}$ for small fullerene films obtained by LECBD [3.

We found that the total particle-particle correlation function of the deposited film has two main peaks corresponding to first and second neighbour distances. Each of these peaks is split, with the first double peak at 1.42 and $1.53 \AA$ : these distances correspond to the 
two different intra-fullerene bond lengths (see Table). The longer distance also corresponds to the inter-fullerene bond length: the correlation function of the sites involved in intermolecular bonds has two single main peaks, the first being centered at $1.52 \AA$. Most fullerenes are three- and fourfold coordinated, although some two-fold and five-fold cages were also observed. This predominance of three- and fourfold coordinated molecules indicates that the $\mathrm{C}_{28}$ cluster has bonding properties similar to those of $\mathrm{C}$ atoms in amorphous systems, which usually contain a mixture of $\mathrm{sp}^{2}$ and $\mathrm{sp}^{3}$ sites 25. This is in agreement with the proposition of Kroto [6] that $\mathrm{C}_{28}$ should behave as a carbon superatom with four preferred active sites (A sites; see Table for definition of site types). We found that A sites have the highest probability (0.37) to form bonds, giving rise to hyperdiamond-like configurations. They are then followed by C (0.11) and B (0.04) sites, in order of decreasing probability. We note that the structural properties of the film deposited in our simulation are remarkably similar to those of a disordered system obtained by subsequent collisions of $\mathrm{C}_{28}$ 's, mimicking gas phase deposition of the clusters [28]. Our findings support early conjectures of Guo et al [5] that $\mathrm{C}_{28}$ solids in a hyperdiamond structure are stable, and are in agreement with the study of Kaxiras et al. [9] on the reactivity of $\mathrm{C}_{28}$.

The proportion of fourfold coordinated atoms and thus of proper $\mathrm{sp}^{3}$ sites is low (10 \%) in the deposited film. Experimentally, from analyses of Raman spectra it has been suggested [3] that films obtained by depositing small fullerenes might be composed of small graphitic islands embedded in a diamond-like matrix. We do not find any evidence of such structures in our deposition energy regime. However we note that the computed vibrational spectra (both within LDA and TB) of free $\mathrm{C}_{28}$ [26] (and $\mathrm{C}_{20}$ [27]) cages are consistent with the reduced Raman spectra calculated from experimental data on small fullerene films. Neither type of spectra exhibit prominent peaks corresponding to graphite Raman active modes (the maximum vibrational frequency [26] for the fullerene cages is about $1500 \mathrm{~cm}^{-1}$ ) and both spectra show peaks centered around 600 and $1200 \mathrm{~cm}^{-1}$. This indicates that the experimentally measured Raman spectra might show direct evidence for the presence of cages in the film, consistent with the observed memory effects. Calculation of the phonon 
density of states of the deposited film is underway and will help clarifying this point [26].

In conclusion, our work shows that using $\mathrm{O}(\mathrm{N})$ methods it is now possible, for certain types of system, to perform TB molecular dynamics simulations on supercells of several thousand atoms for tens of picoseconds. This allows phenomena, previously inaccessible to quantum molecular dynamics, to be studied. In the $\mathrm{O}(\mathrm{N})$-TB molecular dynamics simulations presented in this paper, we have shown that in a given energy range small fullerenes can be assembled on a semiconducting surface to form the first layers of a thin film where the $\mathrm{C}_{28}$ 's constitute the building blocks and act as carbon superatoms, giving rise to hyperdiamondlike configurations. Our results are in agreement with memory effects detected in small fullerene films deposited by LECBD [3] and help clarify the structure of disordered small fullerene films. They also suggest the possibility to synthesize novel new thin films, taking advantage of the many and varied properties of pure and doped fullerene cages. 


\section{REFERENCES}

* galli@eldpa.epfl.ch. To whom correspondence should be addressed.

† Present address: National Renewable Energy Laboratory, Golden Co 80401, USA.

[1] See, e.g., R. F. Service, Science 271, 920 (1996), and G.E. Scuseria, Science 271, 942 (1996).

[2] H. W. Kroto, J. R. Heath, S. C. O'Brien, R. F. Curl and R. E. Smalley, Nature 318, $1744(1985)$.

[3] V. Paillard et al. Phys. Rev. Lett. 71, 4170 (1993); V. Paillard et al. Phys. Rev. B 49, 11433 (1994).

[4] Y. Achiba et al., Materials Science and Engineering B19, 14 (1993).

[5] T. Guo et al, Science 257, 1661 (1992).

[6] W. H. Kroto, Nature 329, 529 (1987).

[7] G. B. Adams, O. F. Sankey, J. B. Page and M. O'Keeffe, Chem. Phys. 176, 61 (1993).

[8] D. M. Bylander and L. Kleinman, Phys. Rev. B 47, 10967 (1993).

[9] E. Kaxiras, L. M. Zeger, A. Antonelli and Y-m Juan, Phys. Rev. B 49, 8446 (1994).

[10] L. M. Zeger, Y-m Juan, E. Kaxiras and A. Antonelli, Phys. Rev. B 52, 2125 (1995).

[11] See, e.g., S.-Y. Qiu, C.Z. Wang, and C.T. Chan, J. Phys. Condensed Matter 6, 9153 (1994) and references therein.

[12] F. Mauri and G. Galli, Phys. Rev. B 50, 4316 (1994); P. Ordejón, D. Drabold, R. Martin and M. Grunbach, Phys. Rev. B 51, 1456 (1995).

[13] J. Kim, F. Mauri and G. Galli, Phys. Rev. B 52, 1640 (1995).

[14] A. Canning, G. Galli, F. Mauri, A. De Vita and R. Car, Comp. Phys. Comm. 94, 89 
(1996).

[15] P. Ordejón, D. A. Drabold, R. M. Martin and S. Ito, Phys. Rev. Lett. 75 , 1324 (1995);

S. Ito, P. Ordejón and R. M. Martin, Phys. Rev. B 53, 2132 (1996).

[16] We adopted the TB Hamiltonian $(\mathcal{H})$ proposed by Xu et al. (J.Phys. Condensed Matter 4, 6047 (1992)) with a cutoff radius of $2.30 \AA$ for the hopping parameters and the two body repulsive potential, and $\mathrm{U}=8 \mathrm{eV}$ for the Hubbard like term. We used the $\mathrm{O}(\mathrm{N})$ formulation of Ref. [13] with three localised functions per atomic site. The localisation regions (LRs) were centered on atomic sites, and contained on average 20 atoms. Tests with larger LRs for clusters of $\mathrm{C}_{28}$ 's and for the substrate used in the deposition experiment gave very similar structural properties. At each molecular dynamics step $\mathcal{H}$ was diagonalised iteratively with a conjugate gradient procedure.

[17] In the deposition simulation we performed 15 iterations per ionic move to diagonalise the TB Hamiltonian, with a time step for the integration of the equations of motion of 10 a.u. The total simulation time was 70 ps.

[18] S.Nosé, Mol. Phys. 52, 255 (1984); W. Hoover, Phys. Rev. A 31, 1695 (1985).

[19] This procedure is similar to that adopted by, e.g., P. C. Weakliem, C. J. Wu and E. A. Carter, Phys. Rev. Lett. 69, 200 (1992).

[20] G. Galli and F. Mauri, Phys. Rev. Lett. 73, 3471 (1994).

[21] H. Xu, D. M. Chen and W. N. Creager, Phys. Rev. Lett. 70, 1850 (1993).

[22] See, e.g., A. Zangwill, Physics at surfaces, Cambridge University Press 1990, page 428.

[23] From our heating experiment, we concluded that the equilibration of a film containing more that $\simeq 50$ fullerenes would become extremely long and eventually unaffordable, due to the low frequency modes associated with the oscillations of the polymer-like structures. 
[24] In the deposited film, the maximum and minimum ratio between the principal moment of inertia are 1.3 and 1.1, respectively and the variations of the maximum and minimum distance within a cluster are 1.54-1.65 and 1.30-1.40 $\AA$, respectively.

[25] J. Robertson, Progress in Solids State Chem. 21, 199 (1991).

[26] P. Ordejón, G. Galli, J. Kim and A. Canning (to be published); the highest vibrational frequency of the $\mathrm{C}_{28}$ cage is 1471 and $1464 \mathrm{~cm}^{-1}$, when computed using a TB and an LDA picture, respectively.

[27] G. Galli, F. Gygi and J.-C. Golaz (to be published).

[28] J. Kim, G. Galli, J. Wilkins and A. Canning (to be published).

[29] R. Car and M. Parrinello, Phys. Rev. Lett. 55, 2471 (1985).

[30] N. Troullier and J. Martins, Phys. Rev. B 43, 1993 (1991).

[31] This work was done as part of the PATP (Parallel Applications Technology Program) joint project between the EPFL and Cray Research. Support is also acknowledged from the Swiss National Science Foundation under Grant No. 20-39528.93 (GG) and from the United States DOE (JK). We thank X. Blase, R. Car, J.-C. Charlier, F. Mauri, P. Ordejón and J. Wilkins for useful discussions. We also thank A. De Vita for support and suggestions with the LDA calculations, performed with a parallel code developed within the PATP. 


\section{TABLES}

\begin{tabular}{|lcc|}
\hline \hline $\mathbf{C}_{\mathbf{2 8}}$ monomer & $\mathrm{O}(\mathrm{N})-\mathrm{TB}$ & LDA \\
\hline A-B bond length $(\AA)$ & $1.44-1.46$ & $1.42-1.45$ \\
A-C bond length $(\AA)$ & $1.40-1.44$ & $1.40-1.44$ \\
C-C bond length $(\AA)$ & $1.47-1.55$ & $1.46-1.57$ \\
\hline $\mathbf{C}_{\mathbf{2 8}}$ dimer & $\mathrm{O}(\mathrm{N})-\mathrm{TB}$ & $\mathrm{LDA}$ \\
\hline A-A bonded $\mathbf{R}_{\mathbf{e q}}(\AA)$ & 1.54 & 1.51 \\
A-C bonded $\mathbf{R}_{\mathbf{e q}}(\AA)$ & 1.46 & 1.51 \\
C-C bonded $\mathbf{R}_{\mathbf{e q}}(\AA)$ & 1.50 & 1.53 \\
A-A bonded $\mathbf{E}_{\mathbf{c o h}}(\mathrm{eV})$ & 3.02 & 3.15 \\
A-C bonded $\mathbf{E}_{\mathbf{c o h}}(\mathrm{eV})$ & 2.13 & 2.48 \\
C-C bonded $\mathbf{E}_{\mathbf{c o h}}(\mathrm{eV})$ & 2.24 & 2.48 \\
\hline $\mathbf{H y y}_{\mathbf{y}}$ & $\mathrm{O}(\mathrm{N})-\mathrm{TB}$ & $\mathrm{LDA}$ \\
\hline Lattice constant $(\AA)$ & 15.85 & 15.78 \\
$\mathbf{E}_{\mathbf{c o h}}(\mathrm{eV})$ & 0.65 & 0.74 \\
$\mathrm{C}_{28}$ - $\mathrm{C}_{28}$ bond length $(\AA)$ & 1.52 & 1.54 \\
\hline \hline
\end{tabular}




\section{TABLE CAPTION}

Comparison between properties of the $\mathrm{C}_{28}$ monomer and dimer and of hyperdiamond obtained from first-principles calculations using the local density functional theory (LDA), and from semi-empirical tight-binding (TB) calculations using the $\mathrm{O}(N)$ method of Ref. [13]. The LDA calculations for hyperdiamond are taken from Ref. [9]. The LDA calculations for the monomer and the dimer were carried out within the pseudopotential-plane-wave formalism [29], using Troullier-Martins pseudopotentials [30] and a kinetic energy cutoff of 30 and 44 Ry (to check for convergence). We used supercells of dimensions $(15.9 \AA)^{3}$ and $(15.9 \AA \times$ $15.9 \AA \times 23.8 \AA$ ) for the monomer and the dimer, respectively. Since the $\mathrm{C}_{28}$ molecule is Jahn-Teller distorted and polarisation effects can be important, we checked for convergence of total energy with respect to cell size and found that in a cell of $(15.9 \AA)^{3}$ the energy was converged up to $0.005 \mathrm{eV}$. The $\mathrm{C}_{28}$ monomer is composed of 12 pentagons and 4 hexagons; atoms at an apex where three pentagons meet are denoted as A sites; atoms on the hexagons are denoted as $\mathrm{B}$ sites if bonded to $\mathrm{A}$ sites, and $\mathrm{C}$ sites otherwise. The $\mathrm{C}_{28}$ molecule has 12 A-B, 24 B-C and 6 C-C bonds. The $\mathrm{C}_{28}$ dimer is composed of two $\mathrm{C}_{28}$ molecules connected by a single bond with equilibrium bond length $\mathbf{R}_{\text {eq }}$ : we considered three cases involving $A$ and $\mathrm{C}$ atoms as bonding sites, since these are the most reactive sites (see text and Ref. [6 9]). Ionic positions of both the monomer and the dimer were fully optimised and the equilibrium rotational configuration of the dimer was the same for both $\mathrm{O}(\mathrm{N})-\mathrm{TB}$ and LDA calculations. The cohesive energy of the dimer was taken to be $\mathbf{E}_{\mathbf{c o h}}=\left(E^{\mathrm{D}}-2 \cdot E^{\mathrm{M}}\right)$, where $E^{\mathrm{D}}$ and $E^{\mathrm{M}}$ are the total energies of the dimer and the monomer, respectively. The cohesive energy of hyperdiamond is with respect to the cohesive energy of diamond. In the $\mathrm{O}(N)$-TB calculation for hyperdiamond both the lattice constant and the atomic position were optimised, whereas in the LDA calculation only the relative position of the $\mathrm{C}_{28}$ 's, assumed to be in a perfect tetrahedral geometry, was optimised [9]. $\mathrm{O}(N)$-TB calculations with 8 and $64 \mathrm{C}_{28}$ 's per cell gave almost identical results for the properties of hyperdiamond reported in the Table. 


\section{FIGURES}

Fig. 1 A snapshot of the full system (4472 atoms) at the end of the molecular dynamics deposition simulation showing the undamaged $\mathrm{C}_{28}$ cages. Red and blue spheres represent atoms belonging to the surface and to the fullerenes, respectively. Bonds between surface atoms and between fullerene atoms are represented as light and dark green, respectively. Note the numerous bonds between the fullerene and the first surface layer causing the local de-reconstruction of sections of the Pandey chains.

Fig. 2 Sections of the system displayed in Fig. 1 (colour scheme as defined in Fig. 1

legend). (A) Top view of the top layer of the substrate showing only the fullerenes directly bonded to the surface: note the random positions of the fullerenes bonded to the surface. (B) top and (C) side view of the top layer of the substrate showing only the fullerenes which are not bonded to the surface: the tendency of the fullerenes to form islands and polymer type structures is clearly visible. 


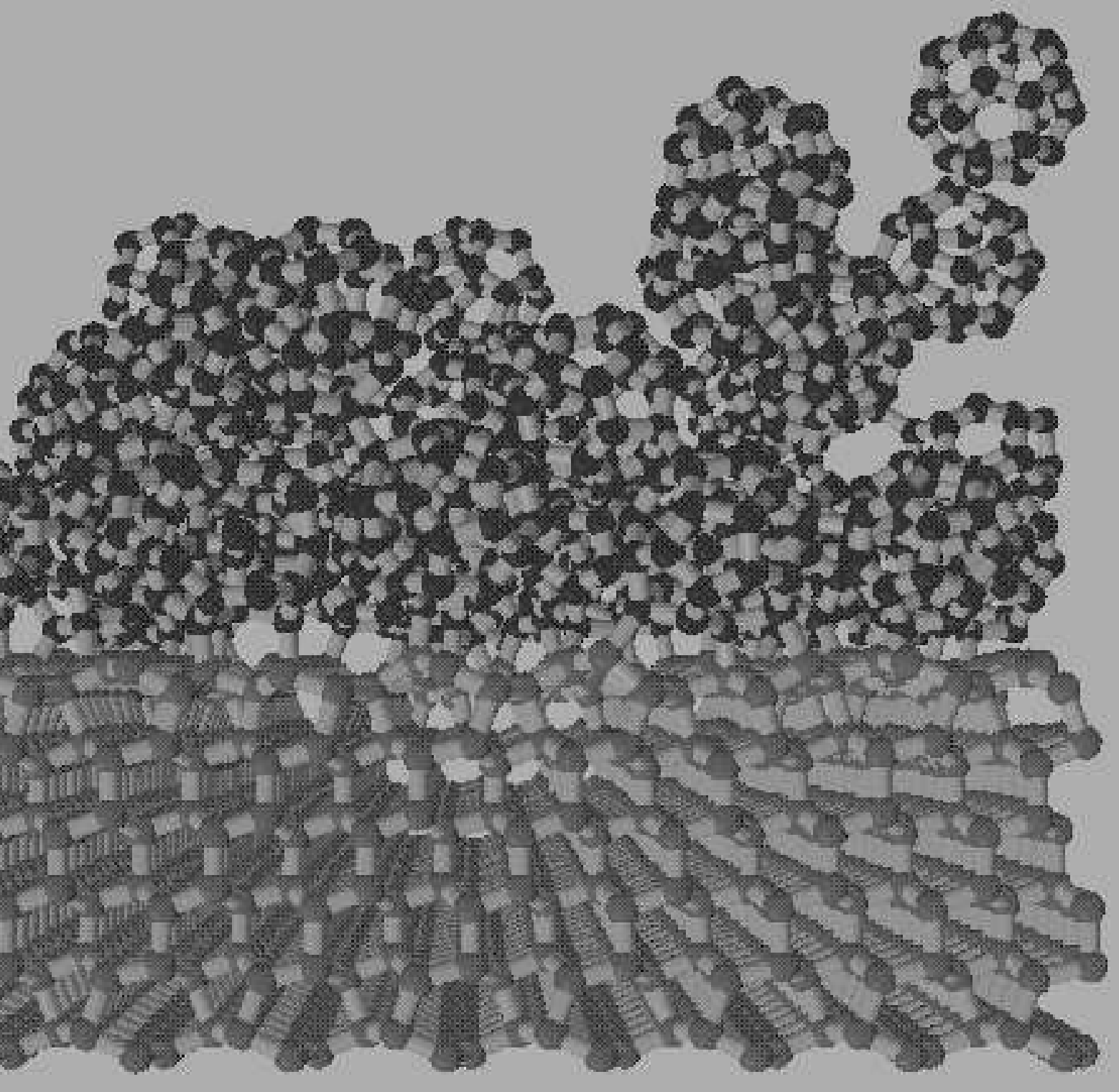


